\title{
Determinación del contenido de zinc en residuos producidos durante el proceso de galvanizado por inmersión
}

\section{Determination of zinc content in waste produced during the immersion galvanizing process}

\author{
SALAZAR-PERALTA, Araceli†*, PICHARDO-SALAZAR, José Alfredo, PICHARDO-SALAZAR, \\ Ulises y CHÁVEZ, Rosa Hilda
}

${ }^{1}$ Tecnológico de Estudios Superiores de Jocotitlán, Carretera Toluca Atlacomulco km 44.8, Ejído de San Juan y San Agustín, Jocotitlán, México.

${ }^{2}$ Centro de Bachillerato Tecnológico Industrial y de Servicios No. 161, Exhacienda la Laguna S/N Barrio de Jesús 2a Sección, San Pablo Autopan, Toluca. Estado de México

${ }^{3}$ Centro de Estudios Tecnológicos Industrial y de Servicios no. 23. Avenida, Del Parque s/n, 52000 Lerma de Villada, México.

${ }^{4}$ Instituto Nacional de Investigaciones Nucleares, Carretera México-Toluca s/n. La Marquesa, Ocoyoacac, 52750, México.

ID $1^{\text {er }}$ Autor: Aracel, Salazar-Peralta / ORC ID: 0000-0001-5861-3748, Researcher ID Thomson: U-2933-2018, CVU CONACYT ID: 300357

ID $1^{\text {er }}$ Coautor: José Alfredo, Pichardo-Salazar / ORC ID: 0000-0002-8939-9921

ID $2^{\text {do }}$ Coautor: Ulises, Pichardo-Salazar / ORC ID: 0000-0002-3758-2038

ID $3^{\text {er }}$ Coautor: Rosa Hilda, Chávez / ORC ID: 0000-0002-2460-3346

DOI: $10.35429 / \mathrm{JCPE} .2020 .22 .7 .1 .8$

Recibido 10 de Enero, 2020; Aceptado 30 de Junio, 2020

\section{Resumen}

En el proceso de galvanizado en caliente se producen remanentes que deben ser removidos para evitar una mala calidad en el recubrimiento del producto. Durante el proceso de galvanizado por inmersión en caliente se obtienen subproductos generados tanto en las tinas como en pailas de galvanizado los cuales pueden ser reutilizados. El objetivo de este estudio fue recuperar Zinc a partir de los subproductos generados durante el proceso de galvanizado por inmersión en caliente. El estudio se hizo caracterizando la composición química, la densidad y cantidad de los residuos generados durante el proceso de galvanizado, los cuales fueron: Zinc recuperado, dross, gota y finos de Zinc. El promedio mensual de los subproductos generados durante un año fue de $6900 \mathrm{~kg}$ de dross, $5800 \mathrm{~kg}$ de gota y $5300 \mathrm{~kg}$ de finos de Zinc. Se obtuvieron $6500 \mathrm{~kg}$ de Zinc recuperado. Se recuperó un $43 \%$ de Zinc. Los resultados fueron favorables para poder llevar a cabo la recuperación del mineral. Se concluye que es necesario seguir trabajando en la recuperación del Zinc, ya que se tendrá un ahorro económico, y además se colaborará con el cuidado del medio ambiente evitando que residuos de Zinc vayan a dar al agua residual.

Determinación de Zinc, Galvanizado por inmersión, Residuos

\begin{abstract}
In the hot galvanizing process, remnants are produced that must be removed to avoid a poor quality in the coating of the product. During the hot dip galvanizing process, by-products are obtained both in the tubs and in galvanizing pails, which can be reused. The objective of this study was to recover Zinc from the byproducts generated during the hot dip galvanizing process. The study was carried out by characterizing the chemical composition, density, and quantity of the by-products generated during the galvanizing process, which were recovered: Zinc, dross, droplet, and Zinc fines. The monthly average of the byproducts generated for one year was $6,900 \mathrm{~kg}$ of dross, $5,800 \mathrm{~kg}$ of drop and $5,300 \mathrm{~kg}$ of zinc fines. $6500 \mathrm{~kg}$ of recovered Zinc were obtained. $43 \%$ Zinc was recovered. The results were favorable to be able to carry out the recovery of the mineral. It is concluded that it is necessary to work on the recovery of Zinc, since there will be economic savings, and it will also collaborate with the care of the environment by preventing Zinc residues from going to wastewater.
\end{abstract}

Zinc Determination, Immersion Galvanized, Wasten

Citación: SALAZAR-PERALTA, Araceli, PICHARDO-SALAZAR, José Alfredo, PICHARDO-SALAZAR, Ulises y CHÁVEZ, Rosa Hilda. Determinación del contenido de zinc en residuos producidos durante el proceso de galvanizado por inmersión. Revista de Energía Química y Física. 2020. 7-23:1-8.

\footnotetext{
* Correspondencia al Autor (Correo Electrónico: araceli.salazar@tesjo.edu.mx)

$\dagger$ Investigador contribuyendo como primer autor
} 


\section{Introducción}

El Zinc es un elemento químico metálico de color blanco con tintes azulados, brillante y blando, es abundante en la corteza terrestre. El Zinc es el cuarto metal en términos de consumo y se encuentra en la naturaleza, en su mayor parte en forma de sulfuro (SZn) mineral denominado blenda o esfalerita, de color caramelo y marmatita de color negruzco. La palabra zinc viene del alemán "zinke" que significa punta en español.

El Zinc es un metal esencial para el desarrollo de la sociedad moderna. Se usa como revestimiento contra la corrosión en el acero, para fabricar componentes de precisión, como material de construcción para producir bronce y caucho.

Los materiales o compuestos de zinc se han utilizado durante siglos, se utiliza en la fabricación de pilas eléctricas, para formar materiales como el latón y para galvanizar el hierro y el acero. La principal aplicación del zinc (cerca del 50\% del consumo anual en el mundo) es el galvanizado del acero que sirve para protegerlo de la corrosión. [1]

El zinc es un metal que se extrae de las minas de sulfuro de zinc y se transforman en zinc metálico mediante uno de estos procesos: reducción térmica, retortas horizontales, electrotermia, ISF (Imperial Smelting Furnace) y electrolisis. Actualmente, el método más usado es el electrolítico con un $85 \%$ de la producción mundial de Zinc.

\section{Antecedentes}

Es probable que el Zinc fuera conocido desde la antigûedad, aunque su producción y su uso fueron ocasionales y su naturaleza ignorada. La primera cita que se tiene de él, la realiza Strabos en el pasaje que escribe a Andriera en Mjsia.

La pieza de Zinc más antigua que se conoce es un ídolo prehistórico hallado en el yacimiento de Dordoch en Transilvania, con un contenido en Zinc del $87,5 \%$ y en Plomo del $11.4 \%$.
Los latones eran conocidos por los romanos por lo menos desde el año 200 a. C. y en la época del Emperador Augusto se preparaban fundiendo en crisol, óxido de zinc, minerales con carbón y cobre; primero se producía óxido de zinc, los valores de este se difundían en el cobre y finalmente incrementando la temperatura para fundir la carga.

Este sistema para fabricar latones era conocido por los alquimistas y su conocimiento para utilizar el Zinc o material que conocían Zinc consiguiendo de esta manera la transmutación de cobre en oro. [2]

La primera vez que se utilizó la palabra Zinc, fue en los escritos de Basilio Valentino, alrededor del siglo XV. A lo largo del siglo XVI, Paracelso aplicó este nombre al metal que hoy se conoce como tal. Por otro lado, se atribuye al alemán Andras Marggraf el primero en usar el término "Zink" para denominar a este metal.

En Asia, Kazwiui, llamado el Plinio de Oriente, que murió hacia el año 600 d. C. indica que el zinc, era conocido en China, y se aplicaba a la fabricación de monedas y espejos.

En la India al parecer era conocido entre los años 100 a 1300 d. C. y probablemente comercializado hacia el siglo XIV.

El primer minero-metalúrgico de la industria del Zinc fue Mahaeaja del Estado de Mewar, la India, el cual en el área de Zawar, explotó las minas y desarrolló una importante industria metalúrgica hacia los años 1380 d. C. Grandes escombreras de residuos, conteniendo Zinc y Plomo, son el testimonio de esta gran industria, que, por el tamaño, debió de ser de gran magnitud. La explotación de estas minas fue alterada en el curso de los años debido a la guerra civil entre los Estados Indios y finalmente abandonadas, hacia 1830. En el año 1940 fueron nuevamente abiertas.

Durante los siglos XVII y XVIII fueron traídas de las Indias Orientales grandes cantidades de Zinc, que era conocido como "estaño indico", "Calaaem", "Tutanego" o "Spiauter". 
Hacia 1620 un barco portugués que traía Zinc de las Indias Orientales fue apresado por los holandeses, y su carga vendida en Paris y otras ciudades bajo el nombre de "Speuter" o "Spialter". Este nombre se latinizó para dar "Speltrum" y de aquí el nombre vulgar de "Spelter" con el que todavía hoy se conoce a los lingotes de Zinc.

En 1745 un barco de la Compañía de las Indias Orientales que venía de Cantón con carga de Zinc, naufragó cerca de Ghotenburg (Suecia); el análisis posterior de estos lingotes, dio el siguiente resultado, $\mathrm{Zn} 98.8 \%$; Fe $0.765 \%$ y Sb $0.245 \%$.

Aproximadamente hacia 1730 pasó de China a Inglaterra el procedimiento metalúrgico para la obtención de Zinc y en 1739 se obtuvo en Inglaterra la primera patente para la obtención metalúrgica del Zinc por destilación. Aproximadamente, entre 1740 y 1743 , se levantó una fundición de Zinc en Bristol, Inglaterra, con una producción de 200 Toneladas al año.

Hasta 1758 solo fueron utilizados óxido de zinc naturales para la obtención de zinc metal, pero a partir de este momento se comenzaron a utilizar los minerales sulfatados, después de una tostación previa.

Hacia 1798 se introdujo la metalurgia del Zinc en Silesia y en 1805 se mejoró el método Ingles, utilizando calaminas como materia prima.

Hasta este momento se había utilizado hornos con retortas horizontales, pero hacia finales del siglo XII se comienzan a utilizar en Corintia retortas verticales.

La pequeña capacidad de estas retortas hizo que el procedimiento fuera abandonado rápidamente, aunque fue incapaz de experimentar un desarrollo comercial, introdujo la idea de las retortas verticales.

Finalmente, en el siglo XX, fue perfeccionando en Bélgica el proceso de obtención de $\mathrm{Zn}$ en retorta horizontal, dando lugar al proceso conocido como "Método Belga". El año 1812 fue importante para la industria del Zinc, pues en él se realizó por primera vez la laminación del Zinc en Lieja. [3], [4]
Hacia 1880 se dieron los primeros pasos en la hidrometalurgia del Zinc, tostando una MENA compleja sulfatada, sulfatizando y disolviendo el sulfato de zinc formado. El sulfato de zinc se cristalizó y se aplicó a la preparación del lingote.

En 1881 se introdujo el horno Hegeler, para realizar la tostación de los minerales sulfatados, sin dejar de mover el material a tostar.

El primer trabajo experimental para la obtención del Zinc electrolítico se realizó en 1881, electrolizando una disolución de sulfato de Zinc, obtenida inhabilitando un mineral tostado, entre un ánodo de plomo y cátodo de cobre o Zinc. La disolución electrolizada fue antes pulverizada y el electrolito agotado fue utilizado para tratar más tostado, o sea prácticamente el mismo esquema del flujo que en el proceso electrolítico actual. Una planta de este tipo se construyó hacia finales de los años 1890, pero fracasó, y esto hizo que la idea de la obtención electrolítica de Zinc se estancara algunos años. [5]

Por el contrario, se desarrolló un procedimiento para la electrólisis del cloruro de zinc. Entre 1896 y 1924 una planta con capacidad de 5-10 toneladas diarias de Zinc, propiedad de Brummer, estuvo en explotación obteniendo Zinc por electrólisis de una disolución de cloruros.

La gran demanda de Zinc durante la segunda guerra mundial revolucionó esta metalurgia, tanto la calidad como la cantidad. Por una parte, permitió utilizar concentraciones de Zinc más puras, con menos impurezas, y por otra parte cooperó en la construcción de nuevas fábricas de Zinc. Al mismo tiempo, se solucionaron los problemas que impedían el desarrollo del método electrolítico, lo cual permitió importarlo en forma comercial.

El método de destilación en continuo se comenzó a considerar en 1830, pero hasta 1925 no fue realizado prácticamente. Aunque los primeros intentos fueron realizados en Alemania e Inglaterra, no tuvieron éxito hasta la puesta en práctica en New Jersey, considerado un avance metalúrgico. [6] 
Actualmente, la tecnología electrolítica es el desarrollo que más tonelaje de metal produce, por su economía y por la calidad del metal. [7], existen multitud de métodos para la obtención metalúrgica del Zinc, pero todos ellos están basados en los procesos ya existentes, derivados fundamentalmente de los últimos descubrimientos físicos y químicos de su mecanización.

Únicamente, y basado en los mismos principios, pero dirigidos en otro sentido, ya que su base está en la metalurgia del plomo, se desarrolló después de la segunda guerra mundial y a partir de 1946 el proceso I.S.F. (Imperial Smelting Furnace), en el cual se obtiene el Zinc en un horno de cuba. [8]

Durante el proceso de galvanizado por inmersión en caliente se obtienen subproductos que pueden ser reutilizados en el proceso de galvanizado, la cantidad promedio que se genera de estos subproductos es un aproximado de 6.43 ton. y 5.75 ton. entre gota y Zinc recuperado, que es considerada como la escoria o desechos industriales de Zinc.

Este es el residuo de un proceso de galvanizado por inmersión en caliente, el cual tiene que ser removido; ya que de no ser así se obtendría una mala calidad del producto en el recubrimiento.

Estos subproductos son generados tanto en las tinas como en pailas de galvanizado, lo cual representa una pérdida significativa del material puro.

El galvanizado es un proceso en el cual una pieza de metal es recubierta con Zinc con el objetivo de darle mejor apariencia, así como evitar la oxidación y la posterior corrosión del metal base, promoviendo mayor duración de la pieza. El presente trabajo tuvo como objetivo estudiar el proceso de galvanizado por inmersión en caliente, así como al final del proceso recuperar el Zinc contenido en el material remanente.
El galvanizado por inmersión es una técnica que se aplica, para llevar a cabo la protección de materiales ferrosos, los cuales son sumergidos en un baño de Zinc electrolítico para realizar el recubrimiento de las piezas. Este proceso se llevó a cabo a una temperatura entre 480 y $510^{\circ} \mathrm{C}$. Durante el proceso de fusión del metal, este comienza a reaccionar con el medio ambiente y con los residuos de material que son galvanizados, dando como resultado la generación de productos secundarios que no son útiles durante el proceso. Dichos subproductos generados son: Zinc recuperado, dross de Zinc y Zinc en gota que es una mezcla principalmente de hierro $(\mathrm{Fe})$ y zinc $(\mathrm{Zn})$.

\section{Metodología}

El proceso de galvanizado por inmersión en caliente, se llevó a cabo de la siguiente manera:

Desengrase. Se utilizó para eliminar contaminantes.

Una vez que las imperfecciones han sido eliminadas de forma mecánica, se procedió a la preparación de la superficie, para ello primero se introdujeron las piezas en baños con agentes tenso activos, la eficacia de los cuales depende de la concentración del baño, la temperatura de este y la duración del tratamiento.

Lavado. Una vez que las piezas han sido desengrasadas se realiza una limpieza para eliminar los restos de aceites, grasas y de los propios componentes del baño de desengrasado con el objetivo de evitar que pasen a etapas posteriores y dificulten tanto el proceso de galvanizado como la valorización de los residuos.

Se sumergió el material en un baño caliente a $95^{\circ} \mathrm{C}$, evitando así que residuos de desengrasante que pudieran haber quedo adheridos pasen a la siguiente etapa del proceso.

Decapado. Cuyo objetivo es eliminar los óxidos procedentes de las operaciones de manufactura o formados por la exposición al medio ambiente. 
El decapado se realizó sumergiendo la pieza en una solución de ácido clorhídrico diluido en agua al $16 \%$ junto con un inhibidor de corrosión, esta solución provoca que solo tenga lugar la disolución del óxido y no la oxidación del hierro base, ya que en caso de que el hierro base se oxidará, la reacción química producirá $\mathrm{H}_{2}$ el cual puede fragilizar las piezas, además, existiría un gasto innecesario de ácido.

En el proceso de galvanizado se utilizan dos ácidos como ácidos de decapado, el ácido clorhídrico $(\mathrm{HCl})$ o el ácido sulfúrico $\left(\mathrm{H}_{2} \mathrm{SO}_{4}\right)$. En este estudio se utilizó $\mathrm{HCl}$.

Lavado. la pieza vuelve a ser enjuagada con agua para neutralizar el ácido clorhídrico. Esta limpieza es necesaria para eliminar los restos de ácido y, sobre todo, de sales de hierro de las etapas anteriores. Es de suma importancia que no lleguen sales de hierro a los baños de Zinc fundido pues, estas, al entrar en contacto con el baño provocan la formación de un residuo sólido que se conoce como matas de zinc, una aleación compuesta por un $95 \%$ de Zinc y un $5 \%$ de hierro.

Esta aleación, además de aumentar el consumo de Zinc en 20g por gramo de sal de hierro que llega al baño, puede llegar a adherirse a la pieza que se está galvanizando provocando la aparición de imperfecciones en la superficie de la misma.

Fluxado. Después del decapado la superficie del material férreo se encuentra en una situación muy activa, por lo que se oxidará rápidamente, para evitar esto, se implementa la etapa de fluxado, la cual evita que la superficie de la pieza se oxide antes de introducirla al baño de Zn, además de eliminar cualquier residuo de agua existente. El fluxado consiste en sumergir la pieza dentro de una solución compuesta de sales de cloruro de zinc $\left(\mathrm{ZnCl}_{2}\right)$ y cloruro de amonio $\left(\mathrm{NH}_{4} \mathrm{Cl}\right)$, a una temperatura de $70^{\circ} \mathrm{C}$ a $80^{\circ} \mathrm{C}$, por aproximadamente 3 minutos, después de los cuales la pieza es extraída de la solución. A este proceso se le conoce como "vía seca", debido a que, una vez extraída la pieza, se procede a realizar una etapa de secado para lograr la cristalización de las sales de galvanizado, formando así una capa protectora, la cual evitará la oxidación de la superficie, además de funcionar como fundente dentro del baño de Zinc (Zn).
Existe otra variante conocida como "vía húmeda", en la cual la etapa posterior de secado es eliminada, en este caso las sales de cloruro de $\mathrm{Zn}$ y amonio se encuentran en la superficie del baño de $\mathrm{Zn}$, de esta forma la pieza tiene que pasar a través de una cubierta de sales para inmediatamente entrar al baño de Zn. Cuando la pieza es extraída, las sales de cloruro de $\mathrm{Zn}$ y amonio son removidas para que la pieza salga por una zona limpia.

Secado. Esta etapa puede omitirse si es que la pieza se encuentra completamente seca después de la etapa de fluxado. Sin embargo, si la pieza lo necesita, se debe realizar un secado previo a la inmersión en el baño de Zn. La etapa de secado suele realizarse exponiendo la pieza al aire durante 5 minutos. Es importante que la pieza se encuentre completamente seca antes de su inmersión en el baño de $\mathrm{Zn}$, ya que de lo contrario la vaporización súbita del agua, produciría una proyección de $\mathrm{Zn}$ fundido por toda el área de trabajo, con las siguientes consecuencias perjudiciales:

- $\quad$ Riesgo de seguridad para los trabajadores.

\section{- $\quad$ Pérdida de Zn.}

Galvanizado. Inmersión en zinc fundido. Una vez realizada las etapas previas de limpieza, se sumerge las piezas en un baño de zinc fundido. La adecuada limpieza de la superficie permite que esta reaccione con el zinc fundido, formándose las capas de $\mathrm{Zn}-\mathrm{Fe}$ de composición variable en la interfase. La parte externa del recubrimiento tendrá la misma composición que el baño si la reacción ha sido controlada adecuadamente.

El Zinc fundido se encuentra a una temperatura de $450^{\circ} \mathrm{C}$ aproximadamente.

La reacción a esta temperatura es extremadamente rápida, siendo los instantes iniciales donde se forma la parte principal del recubrimiento, formado por una capa fina de aleación hierro-zinc. Una vez formada la capa principal, la reacción se vuelve más lenta, disminuyendo el crecimiento del recubrimiento. El tiempo de inmersión en el baño de zinc fundido oscila desde unos pocos segundos para chapas finas y varios minutos para estructurales de gran tamaño y espesor. Dependerá del espesor que se especifique en el acabado. 
Enfriamiento. Al terminar el proceso de galvanizado de la pieza, esta se enfría mediante una impulsión de aire, o sumergiendo la pieza en un baño de agua. $\mathrm{Al}$ sumergir la pieza en agua se enfría rápidamente consiguiendo de algún modo "congelar" el recubrimiento, evitando que las capas de aleación sigan creciendo. [23], [24].

Inspección. Se puede verificar el espesor de la pieza por microscopía o por medio de un instrumento medidor de espesores.

\section{Resultados}

La reacción entre la capa de fundente y el acero, que fue galvanizado, produjo sales de hierro, las cuales formaron dross.

Los subproductos generados durante el proceso de galvanizado fueron Zinc recuperado, dross, gota y finos de Zinc.

\section{Dross}

El dross de Zinc es una mezcla de mineral de Zinc, hierro, óxido de zinc, óxido de hierro y otros minerales en muy bajo porcentaje, obtenidos en el proceso de galvanizado por inmersión en caliente.

En el proceso de galvanizado por inmersión en caliente, se obtiene un subproducto llamado dross, que es considerado como la escoria o desechos industriales de Zinc, este es residuo de un proceso de galvanizado en caliente, el cual tiene que ser removido; ya que de no ser así se obtendría una mala calidad del producto en el recubrimiento. Su contenido del dross es aproximadamente de $96 \%$ de Zinc y $4 \%$ de hierro.

Debido al alto contenido de Zinc en el dross, su desarrollo representa un gran costo para la empresa, y éste debería ser minimizado. Dependiendo de las prácticas de la planta, el consumo de Zinc que aproximadamente va a terminar en el dross está entre $10-35 \%$.

El dross se produce de las siguientes fuentes: Sales de hierro, formadas en la solución de picheado y/o en la tina del fundente base, las cuales son acarreadas por las piezas hacia la tina de galvanizado, donde reaccionan con el Zinc y con el fundente de superficie, formando el dross.

\section{Zinc recuperado}

El Zinc recuperado es el Zinc que se acumula dentro de la paila junto con el dross en el proceso de galvanizado. Este Zinc es recuperado del dross acumulado en la paila, por lo tanto, el Zinc recuperado se vuelve a usar en la paila para continuar con el proceso.

\section{Gota}

Es un subproducto generado en la paila de inducción, en particular como su nombre lo dice, en forma de gotas.

\section{Finos de zinc}

Son un subproducto que se genera en la paila de inducción en forma de polvo. Estos dos subproductos (gota y finos de zinc) salen de la escoriada de la paila de inducción, después este subproducto es colocado en el arnero para separar la gota de los finos.

\section{Agradecimiento}

Al Tecnológico de Estudios Superiores de Jocotitlán por las horas de Investigación asignadas para realizar el Estudio.

\section{Conclusiones}

Este estudio consistió en la determinación de Zinc generado en el proceso de galvanizado por inmersión en caliente, para su recuperación, realizando una serie de análisis cuantitativos para caracterizar los materiales remanentes generados, determinando el porcentaje de Zinc contenido en cada uno de ellos. La tabla 1 Muestra la densidad de cada uno de ellos, la cual se midió por medio del Método del picnómetro.

\begin{tabular}{|l|r|}
\hline Tipo de Residuo & Densidad en $\mathbf{g} / \mathrm{cm}^{\mathbf{3}}$ \\
\hline Zinc recuperado & $7.5 \mathrm{~g} / \mathrm{cm}^{3}$ \\
\hline Dross & $6.38 \mathrm{~g} / \mathrm{cm}^{3}$ \\
\hline Gota & $6.8 \mathrm{~g} / \mathrm{cm}^{3}$ \\
\hline Finos de Zinc & $6.9 \mathrm{~g} / \mathrm{cm}^{3}$ \\
\hline Zinc electrolítico & $7.12 \mathrm{~g} / \mathrm{cm}^{3}$ \\
\hline
\end{tabular}

Tabla 1 Densidad de los residuos obtenidos del proceso de galvanizado por inmersión 
La determinación de la composición química de los residuos, se hizo por medio de Espectroscopía de absorción atómica (por vía húmeda), los resultados fueron favorables para poder llevar a cabo la recuperación del mineral. (Tabla 2).

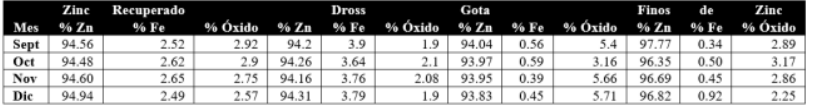

Tabla 2 Composición química promedio mensual, de los residuos obtenidos en el proceso de galvanizado por inmersión, generados de septiembre a diciembre

Para la recuperación de Zinc se investigaron las técnicas de recuperación de minerales y en base a los análisis realizados se concluye que la técnica más recomendable para la recuperación de Zinc es mediante la electrolisis ya que es un proceso favorable en la recuperación del mineral.

El promedio mensual de los subproductos generados durante un año fue de $6900 \mathrm{~kg}$ de dross, $5800 \mathrm{~kg}$ de gota y $5300 \mathrm{~kg}$ de finos de Zinc. Se obtuvieron $6500 \mathrm{~kg}$ de Zinc recuperado. Se recuperó un $43 \%$ de Zinc.

Los resultados fueron favorables para poder llevar a cabo la recuperación del mineral. Se recomienda seguir trabajando en la recuperación del Zinc, ya que se tendrá un ahorro económico, y además se colaborará con el cuidado del medio ambiente evitando que residuos de Zinc vayan a dar al agua residual.

\section{Referencias}

[1] Análisis Comparativo de las Características de Proceso y Rendimiento. Ross Robert. 1988. Pag. 3.

[2] MI MEXICO ES MINERO. Tercera edición. Asociación de Ingenieros de Minas, Metalurgistas y Geólogos de México, A.C. Dr. Rogelio Monreal Saavedra. M.C. Pedro Ignacio Hernández Rábago. Pág. 37.

[3] LATIZA (Asociación Latinoamericana del Zinc). "En la Infraestructura Pública, zinc aplicaciones para el siglo XXI”. Perú, 2008.

[7] MI MEXICO ES MINERO. Tercera edición. Asociación de Ingenieros de Minas, Metalurgistas y Geólogos de México, A.C. Dr. Rogelio Monreal Saavedra. M.C. Pedro Ignacio Hernández Rábago. Pág. 98.
[16] RECUPERACIÓN DEL ZINC DE UNA SOLUCIÓN DE LAVADO DEL PROCESO DE GALVANIZACIÓN. PROYECTO FINAL DE CARRERA. UNIVERSITAT POLITECNICA DE CATALUNYA. Pág. 9.

[19] LATIZA (Asociación Latinoamericana del Zinc). "En la Infraestructura Pública, zinc aplicaciones para el siglo XXI”. Perú, 2008.

[20] Ross Robert B. Handbook of Metal Treatments and Testing [Libro]. - New York: Chapman \& Hall, 1988.

[21] American Galvanizers Association (AGA) "The Inspection of Hot Dip Galvanized Steel Products". 2011 www.galvanizeit.org

[22] National Institute of Steel Detailing and the American Galvanizers Association

"Hot Dip Galvanizing, What We Need to Know" Marzo, 2009 www.nisd.org.

[23] UNIVERSIDAD DE VALLADOLID. Escuela de ingenierías industriales. Descripción del flujograma de una planta de galvanización en caliente, de estructurales. Noriega del Olmo, Ismael. Pág. 41-46.

[24] Universidad politécnica de valencia. Estudio de la recuperación del zinc presente en los baños agotados de decapado procedentes de las industrias de galvanizado de zinc en caliente mediante técnicas electroquímicas. Jordi Carrillo Abad. Pág. 29-35.

[26] Rico et al. / Efecto de la composición química en el comportamiento mecánico de recubrimientos galvanizados por inmersión en caliente: una revisión. Yraima Rico O.1, Edwuin Carrasquero2. Pág. 31.

[27] DISEÑO DE UNA PLANTA DEPURADORA DE EFLUENTES CON ALTO CONTENIDO METÁLICO PARA UNA EMPRESA DE GALVANIZADOS. Universidad de Cádiz. Enrique Navarro Montes. Pág. 34. 
[28] Obtención de recubrimientos de zinc por inmersión en caliente con adición de manganeso y níquel. Universidad nacional autónoma de México. Daniel Hernández Ledesma. Víctor Manuel Hernández Sandoval. Pág 39.

[29] ELECTROQUIMICA CELDAS GALVANICAS, ELECTROLISIS Y ELECTROQUIMICA APLICADA. Pag. 23.

[30] OBTENCIÓN DE POLVOS DE ZINC POR VIA ELECTROLITICA JOHN HENRY PÉREZ MACIAS. Pag. 22 